TRANSACTIONS OF THE

AMERICAN MATHEMATICAL SOCIETY

Volume 352, Number 3, Pages 1021-1038

S 0002-9947(99)02402-2

Article electronically published on March 8, 1999

\title{
DEFORMING A MAP INTO A HARMONIC MAP
}

\author{
DEANE YANG
}

\begin{abstract}
This paper presents some existence and uniqueness theorems for harmonic maps between complete noncompact Riemannian manifolds. In particular, we obtain as a corollary a recent result of Hardt-Wolf on the existence of harmonic quasi-isometries of the hyperbolic plane.
\end{abstract}

\section{INTRODUCTION}

Let $X$ be a complete noncompact Riemannian manifold with Ricci curvature and Sobolev radius (see $\S 6$ for the definition) bounded from below and $Y$ a complete Riemannian manifold with nonpositive sectional curvature. We shall study some situations where a smooth map $f: X \rightarrow Y$ can be deformed continuously into a harmonic map, using a naturally defined flow. The flow used here is not the usual harmonic heat flow, as introduced by Eells-Sampson. We use, instead, a flow introduced by J.P. Anderson [1].

Except for some classical results on linear elliptic partial differential equations, this paper is self-contained and provides a straightforward proof for some existence and uniqueness theorems for harmonic maps. In particular, we obtain as a corollary a recent result of Hardt-Wolf [7] on the existence of harmonic quasi-isometries of hyperbolic space (also, see [16]).

\section{Statement of theorems}

Throughout this paper we shall assume that $X$ is an $n$-dimensional complete noncompact Riemannian manifold with Ricci curvature bounded from below by $-(n-1) \kappa^{2} \leq 0$ and Sobolev radius bounded from below by $2 \rho>0$ (see $\S 6$ for the definition of Sobolev radius) and that $Y$ is an $m$-dimensional complete Riemannian manifold with nonpositive sectional curvature. Also, see $\S 3$ for other definitions.

\section{Definition 1.}

$$
\lambda_{0}(X)=\inf _{f \in C_{0}^{\infty}(X)} \frac{\int_{X}|\nabla f|^{2}}{\int f^{2}} .
$$

Note that $\lambda_{0}(X)>0$ only if $X$ is noncompact and has infinite volume.

Received by the editors July 10, 1997 and, in revised form, December 20, 1997.

1991 Mathematics Subject Classification. Primary 58G30.

I was partially supported by National Science Foundation grant DMS-9200576. Some of the work in this paper was done at l'Institut des Hautes Etudes Scientifiques. I would like thank the staff and the director, Jean-Pierre Bourguignon, for their support and hospitality. I would also like to thank Stephen Semmes, Curt McMullen, and Michael Wolf for helpful discussions. I am grateful to Peter $\mathrm{Li}$ and the referee for their comments on an earlier version of this paper and references to related results. 
Definition 2. We shall say that two maps $u_{1}, u_{2}: X \rightarrow Y$ are within finite $L^{p}$ distance, if the function $d\left(u_{1}(x), u_{2}(x)\right)$ is $L^{p}$-bounded on $X$.

Theorem 1. Assume $\lambda_{0}(X)>0$. Given a harmonic map $u: X \rightarrow Y$, there is no other homotopic harmonic map within finite $L^{p}$ distance of $u$, for any $1<p<\infty$.

Theorem 2. Assume that the sectional curvature of $Y$ is bounded from above by $-K^{2}<0$. Given a strictly nondegenerate harmonic map $u: X \rightarrow Y$, there is no other homotopic harmonic map that is bounded distance away from $u$.

Theorem 3. Assume $\lambda_{0}(X)>0$. Given $p \geq 2$ and a smooth map $u_{0}: X \rightarrow Y$ satisfying

$$
\begin{aligned}
\left\|\Delta u_{0}\right\|_{p} & <\infty, \\
\left\|\partial u_{0}\right\|_{\hat{q}} & <\infty,
\end{aligned}
$$

where

$$
q= \begin{cases}\frac{p n}{n-p}, & 1<p<n, \\ \infty, & p>n,\end{cases}
$$

there exists a homotopic harmonic map $u: X \rightarrow Y$ within finite $L^{p}$ distance of $u_{0}$.

Theorem 4. Given $n, m, \rho, \kappa, K, \eta, E>0$, there exists $\epsilon>0$ such that given $X$ satisfying the assumptions above, $Y$ with sectional curvature bounded from above by $-K^{2}$, and any smooth map $u_{0}: X \rightarrow Y$ satisfying

$$
\begin{aligned}
\eta\left[u_{0}\right] & \geq \eta, \\
\left\|\partial u_{0}\right\|_{\infty} & <E, \\
\left\|\Delta u_{0}\right\|_{\infty} & \leq \epsilon,
\end{aligned}
$$

there exists a homotopic harmonic map $u: X \rightarrow Y$ that is a bounded distance away from $u_{0}$.

Corollary 5. Given $K, E>0$ and $\sigma>1$, there exists $\epsilon>0$ such that given any complete Riemannian manifold $(Y, g)$ with sectional curvature bounded from above by $-K^{2}$ and any Riemannian metric $h$ on $Y$ satisfying

$$
\begin{gathered}
\sigma^{-2} g \leq h \leq \sigma^{2} g, \quad\left\|\nabla_{g} h\right\|_{\infty} \leq E, \\
\left\|g^{i j}\left(\nabla_{i} h_{j k}-\frac{1}{2} \nabla_{k} h_{i j}\right)\right\|_{\infty} \leq \epsilon,
\end{gathered}
$$

there exists a harmonic quasi-isometry $u:(X, h) \rightarrow(X, g)$ homotopic to, and a finite distance from, the identity map.

Let $\mathbf{H}^{n}$ denote hyperbolic $n$-space. By Theorem 4 and Corollary C.4,

Corollary 6. Let $n \geq 2$. Given a harmonic quasi-isometry $u_{0}: \mathbf{H}^{n} \rightarrow \mathbf{H}^{n}$ with boundary map $\hat{u}_{0}$, there exists $\delta>0$ such that given any $(1+\delta)$-quasiconformal $((1+\delta)$-quasisymmetric, if $n=2)$ map $\psi: S^{n-1} \rightarrow S^{n-1}$, the boundary map $\psi \circ \hat{u}_{0}$ : $S^{n-1} \rightarrow S^{n-1}$ extends continuously to a harmonic quasi-isometry $u: \mathbf{H}^{n} \rightarrow \mathbf{H}^{n}$.

Corollary 7. Let $n \geq 2$. There exists $\delta>0$, such that any $(1+\delta)$-quasisymmetric map $\hat{u}: S^{n-1} \rightarrow S^{n-1}$ extends to a harmonic quasi-isometry $u: \mathbf{H}^{n} \rightarrow \mathbf{H}^{n}$. 
Remarks 2.1. Theorems 1 and 3 were first proved by Ding-Wang [5]. The proof presented here is very similar to theirs; the only difference is that by using the flow introduced here, we avoid relying on any past results on harmonic maps. Only the existence and uniqueness of a solution to the Dirichlet problem for a linear elliptic system of partial differential equations is needed here.

Corollary 5 generalizes a result of J. P. Anderson [1].

Corollaries 6 and 7 were first proved by Earle-Fowler in dimension 2 and in all dimensions by Hardt-Wolf [7] in 1993. Another proof appears in a recent paper of Tam-Wan, [16].

The author believes that the condition of bounded energy density is a natural one to impose. A reasonable question to ask whether, given Hadamard manifolds $X$ and $Y$ and a map between the respective boundaries at infinity, there exists an extension of the map to a harmonic map of the interiors with bounded energy density. It is easy, however, to give examples where the answer is no. For example, the map that sends the entire boundary of $X$ to a single point of the boundary of $Y$ has no harmonic extension. A more realistic conjecture appears to be the following:

Conjecture 1. Given Hadamard manifolds $X$ and $Y$, a map between the respective boundaries at infinity can be extended to a harmonic map from $X$ to $Y$ with bounded energy density if and only if the inverse image under the given map between the boundaries of any measure zero set has measure zero.

Note that the condition on the boundary map given in the conjecture is exactly the assumption required for the Douady-Earle extension to exist (see Appendix C).

Remark 2.2. The estimates used in this paper were strongly motivated by work of Coifman-Semmes [4]. Although Coifman and Semmes solve a nonlinear Dirichlet problem on a bounded domain with boundary, the norms they use make it clear that the natural setting for their argument is on the hyperbolic ball. On the hyperbolic ball, one no longer needs to shrink balls as one approaches the boundary, and uniform estimates are even easier to obtain than in the case of the bounded domain.

\section{Definitions Related to A MAP BETWEen RiEmannian MANifolds}

We shall be considering smooth maps $u: X \rightarrow Y$. The differential of $u$ defines a bundle map $\partial u: T_{*} X \rightarrow T_{*} Y$. The energy density of the map $u$ is defined to be

$$
e[u]=|\partial u|^{2} .
$$

Given a bundle $F$ over $Y$ with a connection $\nabla$, we can pull back both the bundle $F$ and the connection $\nabla$ using the map $u$, yielding a bundle $u^{*} F$ over $X$ with connection $u^{*} \nabla$, which we shall usually just denote by $\nabla$, for simplicity.

For example, if $u$ maps all of $X$ to a single point $y \in Y$, then $u^{*} F$ is the trivial bundle $X \times F_{y}$ and $u^{*} \nabla$ is the trivial connection.

The Hessian of $u$ is defined to be a bundle map $\nabla \partial u: S^{2} T_{*} X \rightarrow T_{*} Y$, where given vector fields $v$ and $w$ on $X$,

$$
\nabla_{v} \partial_{w} u=\nabla_{v}\left(\partial_{w} u\right)-\partial_{\nabla_{v} w} u .
$$

The Laplacian is then defined to be

$$
\Delta u=g^{i j} \nabla_{i} \partial_{j} u .
$$

A map $u: X \rightarrow Y$ is defined to be harmonic, if it satisfies

$$
\Delta u=0 .
$$


Definition 3. A map $u: X \rightarrow Y$ is strictly nondegenerate, if

$$
\eta[u]^{2}=\inf _{\substack{x \in X \\ v \in T_{x} X, v \neq 0}}|\partial u(x)|^{2}-|v|^{-2}|v \cdot \partial u(x)|^{2}>0 .
$$

We shall call $\eta[u]$ the nondegeneracy coefficient of $u$.

Definition 4. Two maps $u_{0}, u_{1}: X \rightarrow Y$ are within finite distance of each other, if

$$
\sup _{x \in X} d\left(u_{0}(x), u_{1}(x)\right)<\infty .
$$

Given $1 \leq p \leq \infty$, the maps are within finite $L^{p}$ distance of each other, if the $L^{p}$ norm of $d\left(u_{0}(x), u_{1}(x)\right)$ is finite.

\section{UNIQUENESS OF HOMOTOPIC HARMONIC MAPS}

Given $C^{2}$ homotopic maps $u_{0}, u_{1}: X \rightarrow Y$, we begin by rederiving a well-known formula for $\Delta d\left(u_{0}(x), u_{1}(x)\right)^{2}$ (also, see $[14,15]$ ).

Since $Y$ has nonpositive sectional curvature, any two smooth homotopic maps $u_{0}, u_{1}: X \rightarrow Y$ can be joined by a geodesic homotopy. Therefore, there exists a unique section $v$ of $u_{0}^{*} T_{*} Y$ satisfying

$$
u_{1}=\exp _{u_{0}(x)} v(x)
$$

Given $0 \leq s \leq 1$, let

$$
u_{s}(x)=\exp _{u_{0}(x)} s v(x) .
$$

Let $v_{s}$ be the section of $u_{s}^{*} T_{*} Y$ given by

$$
v_{s}(x)=\frac{\partial}{\partial s} u_{s}(x) \text {. }
$$

Observe that $v_{s}(x), 0 \leq s \leq 1$, is simply the parallel translation of $v(x)$ along the geodesic $\gamma(s)=\exp _{u_{0}(x)} s v(x)$.

Given $x_{0} \in X$ and a unit tangent vector $e \in T_{x_{0}} X$, let $x(t) \in X,-\delta<t<\delta$, be the unit speed geodesic such that $x(0)=x_{0}$ and $x^{\prime}(0)=e$. Let

$$
\Gamma(s, t)=u_{s}(x(t))=\exp _{u_{0}(x(t))} s v\left(u_{0}(x(t))\right)
$$

and denote

$$
\sigma=\frac{\partial}{\partial s}, \quad \tau=\frac{\partial}{\partial t}, \quad S=\Gamma_{*} \sigma, \quad J=\Gamma_{*} \tau
$$

Let $T(s, t)$ satisfy $T(0, t)=J(0, t), \nabla_{\sigma} T=0$. Note that

$$
S(0, t)=v(u(x(t))) \text {. }
$$

Observe that

$$
0=\left[\Gamma_{*} \sigma, \Gamma_{*} \tau\right]=\nabla_{\sigma} J-\nabla_{\tau} S .
$$

Moreover, for each $t$, the curve $\Gamma(\cdot, t)$ is a constant speed geodesic, and therefore $J$ is a Jacobi field along the geodesic $\Gamma(\cdot, t)$, satisfying

$$
\begin{aligned}
J(0, t) & =\Gamma_{*} \tau=\left(u_{0}\right)_{*} x^{\prime}(t), \\
\nabla_{\sigma} J(0, t) & =\nabla_{\sigma} J(0, t)=\nabla_{\tau} v
\end{aligned}
$$

and the Jacobi equation

$$
\nabla_{\sigma} \nabla_{\sigma} J=R(S, J) S
$$


where $R$ is the Riemann curvature tensor on $Y$. Finally, note

$$
\nabla_{e e}^{2} u_{s}=\nabla_{\tau} \partial_{\tau} u_{s}-\partial_{\nabla_{\tau} \tau} u_{s}=\nabla_{\tau} J(s, 0) .
$$

Observe that $d\left(u_{0}(x), u_{1}(x)\right)^{2}=|v|^{2}$, so that

$$
\Delta d\left(u_{0}(x), u_{1}(x)\right)^{2}=2\left(v \cdot \Delta v+|\nabla v|^{2}\right) .
$$

Now consider

$$
v \cdot \nabla_{e e}^{2} u_{s}=S \cdot \nabla_{\tau} J=\frac{\partial}{\partial t}(S \cdot J)-J \cdot \nabla_{\tau} S .
$$

Since $J$ is a Jacobi field,

$$
\frac{\partial^{2}}{\partial s^{2}}(S \cdot J)=S \cdot \nabla_{\sigma} \nabla_{\sigma} J=R(S, J) S \cdot S=0 .
$$

Therefore,

$$
S \cdot J(s, t)=(S \cdot J)(0, t)+s\left(S \cdot \nabla_{\sigma} J\right)(0, t)
$$

So

$$
\begin{aligned}
S \cdot \nabla_{\tau} J(s, t)= & S \cdot \nabla_{\tau} J(0, t)-J \cdot \nabla_{\tau} S(s, t)+J \cdot \nabla_{\tau} S(0, t) \\
& +s\left[\nabla_{\tau} S \cdot \nabla_{\sigma} J(0, t)+S \cdot \nabla_{\tau} \nabla_{\sigma} J(0, t)\right] .
\end{aligned}
$$

On other hand,

$$
\begin{aligned}
\frac{\partial}{\partial s}\left(J \cdot \nabla_{\tau} S\right) & =J \cdot \nabla_{\sigma} \nabla_{\sigma} J+\nabla_{\sigma} J \cdot \nabla_{\sigma} J \\
& =R(S, J) S \cdot J+\left|\nabla_{\sigma} J\right|^{2} .
\end{aligned}
$$

It follows that

$$
J \cdot \nabla_{\tau} S(s, t)-J \cdot \nabla_{\tau} S(0, t)=\int_{0}^{s} R(S, J) S \cdot J+\left|\nabla_{\tau} S\right|^{2} d r
$$

and that

$$
\begin{aligned}
v_{s} \cdot \nabla_{e} u_{s} & =S \cdot \nabla_{\tau} J \\
& =v \cdot \nabla_{e e}^{2} u-\int_{0}^{s} R\left(v_{r}, \partial_{e} u_{r}\right) v_{s} \cdot \partial_{e} u_{r}+\left|\nabla_{e} v_{r}\right|^{2} d r+s\left[\left|\nabla_{e} v\right|^{2}+v \cdot \nabla_{e e}^{2} v\right] .
\end{aligned}
$$

Summing over $e$ ranging over an orthonormal frame of tangent vectors, we get

$$
v_{s} \cdot \Delta u_{s}=v \cdot \Delta u+\frac{1}{2} s \Delta|v|^{2}-\int_{0}^{s} R\left(v_{r}, \partial^{i} u_{r}\right) v_{r} \cdot \partial_{i} u_{r}+\left|\nabla v_{r}\right|^{2} d r
$$

and

$$
\Delta d\left(u_{0}(x), u_{1}(x)\right)^{2}=v_{1} \cdot \Delta u_{1}-v \cdot \Delta u_{0}+\int_{0}^{1} R\left(v_{s}, \partial^{i} u_{s}\right) v_{s} \cdot \partial_{i} u_{s}+\left|\nabla v_{s}\right|^{2} d s .
$$

Theorem 4.1 (Theorem 1). Assume that $\lambda_{0}(X)>0$. Then any two homotopic harmonic maps $u_{0}, u_{1}: X \rightarrow Y$ that are within finite $L^{p}$ distance of each other, for some $1<p<\infty$, are identical. 
Proof. Let $\delta(x)=d\left(u_{0}(x), u_{1}(x)\right)^{2}$. Then $\delta$ is bounded in $L^{p / 2}$ by assumption and $\Delta \delta \geq 0$, by Lemma 4.1. It follows that $\nabla\left(\delta^{p / 4}\right)$ is in $L^{2}$ and that

$$
\begin{aligned}
\int \delta^{p / 2} & \leq \lambda_{0}(X)^{-1} \int\left|\nabla \delta^{p / 4}\right|^{2} \\
& =C \int \delta^{(p-2) / 2}(-\Delta \delta) \\
& \leq 0
\end{aligned}
$$

Remark 1. Theorem 4.1 and its proof hold, if $Y$ has nonpositive sectional curvature.

Theorem 4.2 (Theorem 2). Assume that the sectional curvature of $Y$ is bounded by $-K^{2}<0$. Let $u_{0}: X \rightarrow Y$ be a $C^{2}$ strictly nondegenerate harmonic map. Any $C^{2}$ harmonic map $u_{1}: X \rightarrow Y$ that is homotopic to, and within finite distance of $u_{0}$, is equal to $u_{0}$.

Proof. Given homotopic maps $u_{0}$ and $u_{1}$, there exists a $C^{2}$ section $v$ of $u_{0}^{*} T_{*} Y$ such that $u_{1}=\exp _{u_{0}} v$.

The function $\delta(x)=d\left(u_{0}(x), u_{1}(x)\right)^{2}$ is $C^{2}$. By the generalized maximum principle, Lemma A.2, there exists a sequence $x_{i} \in X$ such that

$$
\lim _{i} \delta\left(x_{i}\right)=\sup _{x \in X} \delta(x), \quad \limsup _{i} \Delta \delta\left(x_{i}\right) \leq 0 .
$$

On the other hand, by (4.1), Lemma B.1, and the strict nondegeneracy of $u_{0}$,

$$
\begin{aligned}
\Delta \delta & \geq K|v|(\tanh K|v|)\left[\left|\partial u_{0}\right|^{2}-|v|^{-2}\left|v \cdot \partial u_{0}\right|^{2}\right] \\
& \geq \eta^{2} \sqrt{\delta} \tanh K \sqrt{\delta} .
\end{aligned}
$$

Therefore,

$$
\begin{aligned}
\sup _{x \in X} K \eta^{2} \sqrt{\delta} \tanh K \sqrt{\delta} & =\lim _{i} K \eta^{2} \sqrt{\delta\left(x_{i}\right)} \tanh K \sqrt{\delta\left(x_{i}\right)} \\
& \leq \limsup _{i} \Delta \delta\left(x_{i}\right) \\
& \leq 0 .
\end{aligned}
$$

We conclude that $\delta$ vanishes identically.

\section{A FLOW FOR MAPS BETWEen RiemanNian MANifoldS}

We shall demonstrate the existence of a harmonic map by constructing a flow of maps that converges to a harmonic map. It is probably possible to use the harmonic map heat flow, but we use a different flow that appears particularly well-suited to maps that have infinite total energy but bounded energy density.

The flow we use is the following: Given a 1-parameter family of maps $u(t)$ : $X \rightarrow Y, 0 \leq t<\infty$, let $v=\partial_{t} u$ denote the velocity field. Note that $v(t)$ is a section of $u(t)^{*} T_{*} Y$. The equation for the flow is

$$
\Delta v-R\left(\partial^{i} u, v\right) \partial_{i} u=-\Delta u
$$

where $R$ is the Riemann curvature of $Y$ and $\Delta$ denotes the Laplacian(s) defined using the naturally induced connections on the corresponding bundles. It is worth 
noting that, in contrast, the Eells-Sampson harmonic heat flow that has been widely studied is given by

$$
v=\Delta u .
$$

The flow used here is motivated by the observation that

$$
\Delta \partial_{t} u-R\left(\partial^{i} u, \partial_{t} u\right) \partial_{i} u=\nabla_{t} \Delta u .
$$

Therefore, if $u(t)$ is a solution for the flow, it satisfies

$$
\nabla_{t} \Delta u(t)=-\Delta u(t) \text {. }
$$

At this point it is worthwhile noting what the role of the nondegeneracy coefficient is. If the sectional curvature of $Y$ is bounded from above by $K$, then

$$
\begin{aligned}
v \cdot R\left(\partial^{i} u, v\right) \partial_{i} u & \geq-K\left(|\partial u|^{2}|v|^{2}-|v \cdot \partial u|^{2}\right) \\
& \geq-K \eta[u]^{2}|v|^{2} .
\end{aligned}
$$

Using this and the assumptions we impose, we will see that the linear differential operator $\Delta-R\left(\partial^{i} u, \cdot\right) \partial_{i} u$ is always invertible and has a bounded inverse. This leads to the existence and uniqueness of the flow for small time, which is proved in $\S 8$.

On other hand, using the generalized maximum principle for complete Riemannian manifolds and local $L^{p}$ bounds for elliptic PDE's, we will obtain a priori bounds for the flow that imply long time existence and convergence to a harmonic map as $t \rightarrow \infty$. The details are presented in $\S 7$.

Remark 5.2. The flow defined above was introduced in the doctoral dissertation of J. P. Anderson [1], who used it to deform a quasi-isometry between closed Riemannian manifolds into a harmonic diffeomorphism.

\section{BASIC ANALYTIC DEFinitions AND ESTIMATES}

The distance between two points $x$ and $y$ on a Riemannian manifold $X$ will always be denoted $d(x, y)$. A geodesic ball of radius $r$ centered at $p$ will be denoted $B(p, r)$. Let $\widetilde{B}(p, r)$ denote the universal cover of $B(p, r)$ and $\widehat{B}(p, r) \subset \widetilde{B}(p, r)$ be the ball of radius $r$ centered at one of the inverse images of $p$, using the pullback Riemannian metric. Let $\phi_{p}: \widehat{B}(p, r) \rightarrow B(p, r)$ denote the canonical projection map.

Let $E$ be a vector bundle over $X$ with an inner product. Given a section $v$ of $E$, $1 \leq p<\infty$, we shall use the following "local" $L^{p}$ norms:

$$
\|v\|_{\hat{p}}=\sup _{x \in X}\left(\int_{\widehat{B}(x, \rho)}\left|v \circ \phi_{x}\right|^{p}\right)^{1 / p} .
$$

As mentioned before, the idea of using norms like this is motivated by a similar approach used by Coifman-Semmes [4] to solve a Dirichlet problem for a nonlinear elliptic PDE on a bounded domain. We derive some basic properties of these norms below.

Recall the Bishop-Gromov volume comparison theorem (see [3]):

Lemma 6.1. Given a complete Riemannian manifold $X$ with Ricci curvature bounded from below by $-(n-1) \kappa^{2}, p \in X$, and $0<r<R$,

$$
\frac{\operatorname{vol}(\widehat{B}(p, R))}{\operatorname{vol}(\widehat{B}(p, r))} \leq \frac{v_{-\kappa^{2}}(R)}{v_{-\kappa^{2}}(r)}
$$


where $v_{-\kappa^{2}}(r)$ is the volume of a geodesic ball with radius $r$ in the space of constant sectional curvature $-\kappa^{2}$.

Definition 5. Given a complete Riemannian manifold $X$ with Ricci curvature bounded from below by $-(n-1) \kappa^{2} \leq 0$ and $x \in X$, we define the Sobolev radius $\rho_{S}(x)$ to be the largest $r>0$ such that

$$
\operatorname{vol}(\widehat{B}(x, r)) \geq \frac{1}{2} v_{-\kappa^{2}}(r) .
$$

This definition is motivated by the theorem of Gromov (see [3]), which gives a lower bound on the isoperimetric constant and therefore an upper bound on the Sobolev constant on the domain $\widehat{B}\left(x, \rho_{S}(x)\right)$.

Recall that we always assume that the Ricci curvature of $X$ is bounded from below by $-(n-1) \kappa^{2}$ and that the Sobolev radius is always bounded from below by $2 \rho>0$. As a consequence, we obtain the following key lemmas:

Corollary 6.2. There exists $N(n, \kappa)>0$ such that given any $x \in X$, there exist $x_{1}, \ldots, x_{N} \in X$ such that

$$
\widehat{B}(x, 2 \rho) \subset \widehat{B}\left(x_{1}, \rho\right) \cup \cdots \cup \widehat{B}\left(x_{N}, \rho\right) .
$$

Corollary 6.3. Given any locally integrable function $f: X \rightarrow R$ and $x \in X$,

$$
\int_{\widehat{B}(x, 2 \rho)} f \circ \phi_{x} \leq N \sup _{y \in X} \int_{\widehat{B}(y, \rho)} f \circ \phi_{x} .
$$

In particular, for any locally $L^{p}$ function $f$ and $x \in X$,

$$
\left(\int_{\widehat{B}(x, 2 \rho)}|f|^{p}\right)^{1 / p} \leq N^{1 / p}\|f\|_{\hat{p}} .
$$

Also, we have the following Sobolev inequality [3]:

Lemma 6.4. There exists a constant $A$, depending only on the dimension $n$, the lower bound $-(n-1) \kappa^{2}$ of Ricci curvature, and the lower bound $2 \rho$ of the Sobolev radius, such that given any smooth compactly supported function $f$ on $X$, the following inequality holds:

$$
\left(\int|f|^{\frac{2 n}{n-2}}\right)^{\frac{n-2}{n}} \leq A \int|\nabla f|^{2}+f^{2} .
$$

The key analytic estimate we will use is obtained using Lemma 6.4 and a classical technique known as Moser iteration. The version we use is taken from Appendix C of [17].

Lemma 6.5. Let $E$ be a vector bundle over $X$ with an inner product and a compatible connection. Let $R: E \rightarrow E$ be a symmetric linear bundle map and

$$
K_{-}(x)=\min \left(0, \inf _{v \in E_{x} \backslash\{0\}} \frac{v \cdot R(x) v}{|v|^{2}}\right) .
$$


Given $2 \leq p, q \leq \infty$ and $n / 2<s \leq \infty$, there exists $C>0$ such that given sections $v$ of $E$ and $f$ of $T_{*} X \otimes E$ that satisfy

$$
\begin{aligned}
\|v\|_{\hat{p}} & <\infty, \\
\|f\|_{\hat{q}} & <\infty, \\
\left\|K_{-}\right\|_{\infty} & <\infty, \\
-\nabla^{i} \nabla_{i} v+R v & =\nabla \cdot f,
\end{aligned}
$$

then if $p<q<n$,

$$
\|v\|_{\hat{r}} \leq C\left[\left(1+\left\|K_{-}\right\|_{\infty}\right)^{\frac{n}{2}\left(\frac{1}{p}-\frac{1}{r}\right)}\|v\|_{\hat{p}}+\|f\|_{\hat{q}}\right]
$$

where

$$
r=\frac{q n}{n-q}
$$

and if $q>n$,

$$
\|v\|_{\infty} \leq C\left[\left(1+\left\|K_{-}\right\|_{\infty}\right)^{\frac{n}{2 p}}\|v\|_{\hat{p}}+\|f\|_{\hat{q}}\right] .
$$

\section{A PRIORI ESTIMATES FOR THE HARMONIC MAP FLOW}

We first observe that $\Delta u(t)$ decays exponentially.

Lemma 7.1. Let $u(t): X \rightarrow Y, 0 \leq t \leq T$, be a 1-parameter family of maps satisfying

$$
-\Delta \partial_{t} u(t)+R\left(\partial^{i} u(t), \partial_{t} u(t)\right) \partial_{i} u(t)=\Delta u(t) .
$$

Then

$$
|\Delta u(t)|=|\Delta u(0)| e^{-t} .
$$

Lemma 7.2. Assume $\lambda_{0}(X)>0$. Given $p \geq 2$, there exists $C>0$ such that the following holds: Given a smooth map $u: X \rightarrow Y$ such that $\|\Delta u\|_{p}<\infty$ and an $L^{p}$ bounded section $w$ of $u^{*} T_{*} Y$, there exists a unique $L^{p}$-bounded section $v$ of $u^{*} T_{*} Y$ satisfying

$$
-\Delta v+R\left(\partial^{i} u, v\right) \partial_{i} u=w .
$$

Moreover, $v$ satisfies the following estimates:

$$
\begin{aligned}
\|v\|_{p} & \leq C\|w\|_{p}, \\
\|\nabla v\|_{\hat{q}} & \leq C\|w\|_{p},
\end{aligned}
$$

where

$$
q= \begin{cases}\frac{p n}{n-p}, & p<n \\ \infty, & p>n\end{cases}
$$

Proof. First, note that

$$
v \cdot w=v \cdot(-\Delta v)+v \cdot R\left(\partial^{i} u, v\right) \partial_{i} u \geq v \cdot(-\Delta v) .
$$


Therefore,

$$
\begin{aligned}
\int|v|^{p} & \leq \lambda_{0}^{-1} \int\left|\nabla\left(|v|^{p / 2}\right)\right|^{2} \\
& \leq \frac{p^{2}}{4(p-1) \lambda_{0}} \int|v|^{p-2}|\nabla v|^{2}+(p-2)|v|^{p-4}|v \cdot \nabla v|^{2} \\
& =C \int|v|^{p-2} v \cdot(-\Delta v) \\
& \leq C \int|v|^{p-2} v \cdot w \\
& \leq C\|v\|_{p}^{p-1}\|w\|_{p} .
\end{aligned}
$$

This proves the first inequality and the uniqueness of $v$.

Next, observe that using the equation (7.1), we get

$$
\|\nabla v\|_{\hat{2}} \leq C\|v\|_{\hat{2}} \leq C\|v\|_{\hat{p}} \leq C\|w\|_{\hat{p}}
$$

Differentiating and manipulating (7.1), we obtain

$$
\begin{aligned}
-\nabla^{j} \nabla_{j} \nabla_{i} v^{\alpha}- & R^{\alpha}{ }_{\beta \gamma \delta} \partial_{i} u^{\gamma} \partial_{j} u^{\delta} \nabla^{j} v^{\beta}+\widehat{R}_{i}^{j} \nabla_{j} v^{\alpha} \\
& =-\nabla^{j}\left(R^{\alpha}{ }_{\beta \gamma \delta} \partial_{j} u^{\gamma} \partial_{i} u^{\delta} v^{\beta}\right)+\nabla_{i}\left(R^{\alpha}{ }_{\beta \gamma \delta} \partial^{p} u^{\beta} \partial_{p} u^{\delta} v^{\gamma}+w\right) .
\end{aligned}
$$

The bound for $\nabla v$ now follows from the local $L^{2}$ bound on $\nabla v$ and Lemma 6.5. When applying Lemma 6.5, note that we can throw away the term containing the curvature of $Y$, because it is positive definite.

To obtain existence, consider the equation on a sequence of domains with smooth boundary in $X$ that exhausts $X$. On each domain the positivity of the potential implies the existence and uniqueness of a solution with Dirichlet boundary conditions, i.e. boundary value equal to zero. The estimates imply that on any compact domain this sequence of solutions has a subsequence that converges uniformly to a solution. Using a diagonal argument, we obtain a subsequence that converges on any compact subset of $X$ to a global solution of the equation.

Integrating the estimates above yields

Lemma 7.3. Assume $\lambda_{0}(X)>0$. Given $1<p<\infty$, let $C>0$ and $q>n$ be as given by Lemma 7.2. Let $u(t): X \rightarrow Y, 0 \leq t<T$, be a 1-parameter family of maps satisfying

$$
\begin{aligned}
\|v(t)\|_{p} & <\infty, \\
\|\Delta u(t)\|_{p} & <\infty, \\
-\Delta v(t)+R\left(\partial^{i} u(t), v(t)\right) \partial_{i} u(t) & =\Delta u(t),
\end{aligned}
$$

where $v(t)=\partial_{t} u(t)$. Then the following estimates hold for any $0 \leq t<T$ :

$$
\begin{aligned}
\|\Delta u(t)\|_{p} & =\|\Delta u(0)\|_{p} e^{-t}, \\
\|d(u(t), u(0))\|_{p} & \leq C\|\Delta u(0)\|_{p}\left(1-e^{-t}\right), \\
\|\partial u(t)\|_{\hat{q}} & \leq\|\partial u(0)\|_{\hat{q}}+C\|\Delta u(0)\|_{p}\left(1-e^{-t}\right) .
\end{aligned}
$$

With an $L^{\infty}$ bound on $\Delta u(t)$, it's necessary to assume a lower bound on the nondegeneracy of $u(t)$. 
Lemma 7.4. There exists $C>0$ such that the following holds: Let $Y$ have sectional curvature bounded from above by $-K^{2}$. Given a map $u: X \rightarrow Y$ with nondegeneracy coefficient $\eta=\eta[u]$ and a bounded smooth section $w$ of $u^{*} T_{*} Y$, there exists a unique bounded smooth section $v$ of $u^{*} T_{*} Y$ satisfying

$$
-\Delta v+R\left(\partial^{i} u, v\right) \partial_{i} u=w .
$$

Moreover, $v$ satisfies the following estimates:

$$
\begin{aligned}
\|v\|_{\infty} & \leq\left(\frac{K}{\eta}\right)^{2}\|w\|_{\infty} \\
\|\nabla v\|_{\infty} & \leq C\left[1+\left(\frac{K}{\eta}\right)^{2}\right]\|w\|_{\infty} .
\end{aligned}
$$

Proof. Uniqueness of $v$ follows by applying (5.1) and the generalized maximum principle, Lemma A.2, to the elliptic inequality satisfied by $|v|^{2}$. The maximum principle also yields the bound on $\|v\|_{\infty}$. The $L^{\infty}$ bound on $\nabla v$ follows as in the proof of Lemma 7.2 by applying Lemma 6.5 to $(7.2)$.

The existence of a solution $v$ follows by the same argument as in the proof of Lemma 7.2.

Integrating the estimates in Lemma 7.4 yields

Lemma 7.5. There exists $C>0$ such that the following holds: Given $\eta>0$, let $u(t): X \rightarrow Y, 0 \leq t<T$, be a 1-parameter family of maps satisfying

$$
\begin{aligned}
\eta[u(t)] & \geq \eta, \\
\|\partial u(t)\|_{\infty} & <\infty, \\
\|v(t)\|_{\infty} & <\infty, \\
\|\Delta u(t)\|_{\infty} & <\infty, \\
-\Delta v(t)+R\left(\partial^{i} u(t), v(t)\right) \partial_{i} u(t) & =\Delta u(t),
\end{aligned}
$$

where $v(t)=\partial_{t} u(t)$. Then the following estimates hold for any $0 \leq t<T$ :

$$
\begin{gathered}
\|\Delta u(t)\|_{\infty}=\|\Delta u(0)\|_{\infty} e^{-t} \\
\left\|\nabla_{t} \partial u(t)\right\|_{\infty} \leq C \eta^{-2}\|\Delta u(0)\|_{\infty} e^{-t} \\
\|\partial u(t)\|_{\infty} \leq\|\partial u(0)\|_{\infty}+C \eta^{-2}\|\Delta u(0)\|_{\infty}\left(1-e^{-t}\right) \\
\eta[u(t)]^{2} \geq \eta[u(0)]^{2}-C \eta^{-2}\left(\|\partial u(0)\|_{\infty}+\|\Delta u(0)\|_{\infty}\right)\|\Delta u(0)\|_{\infty}\left(1-e^{-t}\right) .
\end{gathered}
$$

Proof. First, (7.4) follows directly from Lemma 7.1, while (7.5) follows from Lemma 7.4 and the observation that

$$
\nabla_{t} \partial u=\nabla v .
$$

The estimate (7.6) follows from integrating (7.5). Finally, to prove (7.7), given a section $w$ of $u(t) * T_{*} Y$, let $w\left(t^{\prime}\right) \in \Gamma\left(u\left(t^{\prime}\right)^{*} T_{*} Y\right), 0 \leq t^{\prime} \leq t$, satisfy

$$
\begin{aligned}
w(t) & =w, \\
\nabla_{t^{\prime}} w & =0 .
\end{aligned}
$$


Then

$$
\begin{aligned}
\frac{\partial}{\partial t^{\prime}}\left(|\partial u|^{2}|w|^{2}-|w \cdot \partial u|^{2}\right) & =2\left(|w|^{2} \partial u \cdot \nabla_{t^{\prime}} \partial u-(w \cdot \partial u) \cdot\left(w \cdot \nabla_{t^{\prime}} \partial u\right)\right) \\
& \geq-C \eta^{-2}|w|^{2}|\nabla u|\|\Delta u(0)\|_{\infty} e^{-t^{\prime}} .
\end{aligned}
$$

Integrating this and taking the infimum over all sections $w$, we get

$$
\eta[u(t)]^{2} \geq \eta[u(0)]^{2}-C \eta^{-2}\left(\|\partial u(0)\|_{\infty}+\|\Delta u(0)\|_{\infty}\right)\|\Delta u(0)\|_{\infty}\left(1-e^{-t}\right) .
$$

\section{EXISTENCE THEOREMS FOR THE HARMONIC MAP FLOW}

We begin with existence of the flow for small time:

Theorem 8.1. Let $u_{0}: X \rightarrow Y$ be a map satisfying

$$
\left\|\partial u_{0}\right\|_{\infty},\left\|\Delta u_{0}\right\|_{\infty}<\infty
$$

and

$$
\eta\left[u_{0}\right]>0
$$

Then there exists $T>0$ and a unique 1-parameter family of maps $u: X \times[0, T) \rightarrow Y$ and $v \in \Gamma\left(u^{*} T_{*} Y\right)$ satisfying the harmonic map flow:

$$
\begin{aligned}
\nabla_{t} u & =v, \\
\|v\|_{\infty} & <\infty, \\
-\Delta v+R\left(\partial^{i} u, v\right) \partial_{i} u & =\Delta u, \\
u(0) & =u_{0} .
\end{aligned}
$$

Moreover, if

$$
\limsup _{t \rightarrow T} \eta[u(t)]>0
$$

then the flow extends beyond $t=T$.

Proof. The existence and uniqueness of a solution for sufficiently small $T>0$ follows from a straightforward application of the usual existence and uniqueness theorem for an ODE on a Banach manifold. We sketch the argument.

Given $C>0$, the space of maps $u: X \rightarrow Y$ satisfying

$$
\begin{aligned}
\eta[u] & >C^{-1}, \\
\left\|d\left(u_{0}, u\right)\right\|_{\infty} & <C, \\
\|\partial u\|_{\infty} & <C, \\
\|\Delta u\|_{\infty} & <C
\end{aligned}
$$

is an open Banach manifold $\mathcal{B}$. We leave to the reader the exercise of constructing coordinate charts for $\mathcal{B}$ and verifying that given $u \in \mathcal{B}$, the tangent space $T_{u} \mathcal{B}$ consists of sections $v$ of $u^{*} T_{*} Y$ satisfying

$$
\|v\|_{\infty},\|\nabla v\|_{\infty},\|\Delta v\|_{\infty}<\infty .
$$

Let $F$ be the section of $T_{*} \mathcal{B}$ where $v=F(u)$ is the unique section of $u^{*} T_{*} Y$ satisfying

$$
\Delta v-R\left(\partial^{i} u, v\right) \partial_{i} u=-\Delta u \text {. }
$$


By Lemma 7.4, $F$ is a well-defined differentiable section of $T_{*} \mathcal{B}$. By the existence and uniqueness theorem for ODE's on a Banach manifold [12], there exists for sufficiently small $T>0$ a unique solution $u:[0, T) \rightarrow \mathcal{B}$ of

$$
\frac{d u}{d t}=F(u) .
$$

If there exists $\eta>0$ such that

$$
\eta[u(t)] \geq \eta
$$

for all $0 \leq t<T$, then the estimates obtained in Lemmas 7.4 and 7.5 imply that $u(t) \rightarrow u(T) \in \mathcal{B}$, for $C$ suitably large. Then the local existence theorem implies that the solution can be extended beyond $t=T$.

Theorem 8.2 (Theorem 4). Given manifold $X, Y$, and $E, \eta>0$, there exists $\epsilon>$ 0 , such that if $u_{0}: X \rightarrow Y$ satisfies

$$
\begin{aligned}
\left\|\partial u_{0}\right\|_{\infty} & \leq E, \\
\eta\left[u_{0}\right] & \geq \eta, \\
\left\|\Delta u_{0}\right\|_{\infty} & <\epsilon,
\end{aligned}
$$

then there exists a unique homotopic harmonic map $u_{1}: X \rightarrow Y$ within finite distance of $u_{0}$.

Proof. By Theorem 8.1, there exists a harmonic map flow $u(t): X \rightarrow Y$ with $u(0)=u_{0}$. By Lemma 7.5, if $\epsilon>0$ is chosen sufficiently small, then $\eta[u(t)] \geq \eta / 2$ for any $t$. Therefore, the flow must exist for all $t>0$. The bounds given in Lemma 7.4 imply that as $t \rightarrow \infty$, the map $u(t)$ converges to a limiting map $u_{\infty}$. Moreover, the limiting map must be harmonic and have bounded distance from $u_{0}$.

In the same way, Lemma 7.3 leads to the following

Theorem 8.3 (Theorem 3). Assume that

$$
\lambda_{0}(X)>0 .
$$

Given $2 \leq p<\infty$, let

$$
q= \begin{cases}\frac{p n}{n-p}, & 1<p<n, \\ \infty, & p>n .\end{cases}
$$

Let $u_{0}: X \rightarrow Y$ be a smooth map satisfying

$$
\begin{gathered}
\left\|\partial u_{0}\right\|_{\hat{q}}<\infty, \\
\left\|\Delta u_{0}\right\|_{p}<\infty .
\end{gathered}
$$

Then there a unique 1-parameter family of maps $u: X \times[0, \infty) \rightarrow Y$ and $v \in$ $\Gamma\left(u^{*} T_{*} Y\right)$ satisfying:

$$
\begin{aligned}
\nabla_{t} u & =v, \\
\|v\|_{p} & <\infty, \\
-\Delta v+R\left(\partial^{i} u, v\right) \partial_{i} u & =\Delta u, \\
u(0) & =u_{0} .
\end{aligned}
$$


Moreover, there exists a smooth limit

$$
u_{\infty}=\lim _{t \rightarrow \infty} u(t)
$$

that is a harmonic map and is within finite $L^{p}$ distance of the map $u_{0}$.

What is not obvious is that given two Hadamard manifolds $X$ and $Y$, whether there exists any nontrivial map $u_{0}$ that has $\Delta u_{0}$ bounded in $L^{p}$ for some $p$. This is, in fact, unlikely to occur in the general situation. Understanding when this is possible would extend the results of Li-Tam to Hadamard manifolds.

\section{Appendix A. A generalized maximum Principle for COMPlete \\ RIEMANNIAN MANIFOLDS}

Lemma A.1. Given $\kappa, r>0$, there exists $C>0$ such that for any complete Riemannian manifold with Ricci curvature bounded from below by $-(n-1) \kappa^{2}, p \in X$, there exists a compactly supported function $\chi$ on $\widehat{B}(p, 2 r)$ such that the following hold:

$$
\begin{aligned}
\chi(x) & =1, x \in \widehat{B}(p, r), \\
\|\nabla \chi\|_{\infty} & \leq C, \\
\Delta \chi(x) & \leq C .
\end{aligned}
$$

Proof. A version of the Bishop-Gromov comparison inequality states that the function $\rho(x)=d(p, x)$ satisfies

$$
\Delta \rho \leq(n-1) \kappa \operatorname{coth} \kappa \rho
$$

in a generalized sense. This is easily proven whenever there is a unique minimal geodesic joining $p$ to $x$. If there is not, then one simply observes that in a neighborhood of $x, \rho$ can be represented as the minimum of a finite number of smooth functions that satisfy the inequality.

It suffices to set $\chi(x)=\psi(\rho(x))$, where $\psi$ is a suitably chosen smooth compactly supported function on the real line.

Lemma A.2 (Omori-Yau maximum principle, [13, 18], also see [2]). Given a complete Riemannian manifold $X$ with Ricci curvature bounded from below and any $C^{2}$ function $f: X \rightarrow \mathbf{R}$ that is bounded from above, there exists a sequence of points $x_{k} \in X$ such that

$$
\begin{aligned}
\lim _{k} f\left(x_{k}\right) & =\sup f, \\
\lim _{k}\left|\nabla f\left(x_{k}\right)\right| & =0, \\
\limsup _{k} \Delta f\left(x_{k}\right) & \leq 0 .
\end{aligned}
$$

Proof. It suffices to prove that there exists a constant $C>0$ such that given any $C^{2}$ function $f$ that is bounded from above and $\epsilon>0$, there exists $x \in X$ such that

$$
\begin{aligned}
f(x) & \geq \sup f-\epsilon, \\
|\nabla f(x)| & \leq C \epsilon, \\
\Delta f(x) & \leq C \epsilon .
\end{aligned}
$$


Given $\epsilon>0$ and a $C^{2}$ function $f$ that is bounded from above, there exists $x^{\prime} \in X$ satisfying $f\left(x^{\prime}\right) \geq \sup f-\epsilon$. Consider the function $\hat{f}: \widehat{B}\left(x^{\prime}, 2\right) \rightarrow \mathbf{R}$, where

$$
\hat{f}(z)=f\left(\phi_{x^{\prime}}(z)\right)-\epsilon[1-\chi(z)]
$$

where $\chi$ is the function given by Lemma A.1. Note that $\hat{f}$ must achieve a maximum at some $z^{\prime} \in B_{0}$. Therefore,

$$
\begin{aligned}
& \nabla \hat{f}\left(z^{\prime}\right)=0, \\
& \Delta \hat{f}\left(z^{\prime}\right) \leq 0 .
\end{aligned}
$$

Therefore, (A.1) hold if $x=\phi_{x^{\prime}}\left(z^{\prime}\right)$.

\section{APPENDIX B. LOWER BOUNDS FOR THE INDEX OF A GEODESIC}

Let $Y$ be a complete Riemannian manifold with strictly negative curvature. Let $\gamma:[0,1] \rightarrow Y$ be a constant speed geodesic. Let $T$ be a nonzero tangent vector at $\gamma(0)$. We want to minimize the quantity

$$
E[J]=\int_{0}^{1} R\left(\gamma^{\prime}, J\right) \gamma^{\prime} \cdot J+\left|\nabla_{\gamma^{\prime}} J\right|^{2} d s
$$

over all Jacobi fields $J$ along $\gamma$ such that $J(0)=T$. Since the functional $E$ is a positive definite quadratic function on a finite dimensional affine space, there is a unique minimum, whose value is calculated below. Computations like this were introduced by Jäger-Kaul [8, 9] and Jost-Karcher [10, 11] to study the uniqueness of solutions to the Dirichlet problem for harmonic maps.

If $J$ is the minimizing Jacobi field, then given any Jacobi field $\dot{J}$ along $\gamma$ such that $\dot{J}(0)=0$,

$$
\begin{aligned}
0 & =E^{\prime}[J] \dot{J} \\
& =2 \int_{0}^{1} R\left(\gamma^{\prime}, J\right) \gamma^{\prime} \cdot \dot{J}+J^{\prime} \cdot \dot{J}^{\prime} d s \\
& =\left.2 \dot{J} \cdot J^{\prime}\right|_{0} ^{1} \\
& =2 \dot{J} \cdot J^{\prime}(1) .
\end{aligned}
$$

Therefore, $J^{\prime}(1)=0$ and

$$
\begin{aligned}
& \inf _{\substack{J \text { Jacobi } \\
J(0)=T}} \int_{0}^{1} R\left(\gamma^{\prime}, J\right) \gamma^{\prime} \cdot J+\left|\nabla_{\gamma^{\prime}} J\right|^{2} d s \\
& =\inf _{\substack{J \text { Jacobi } \\
J(0)=T, J^{\prime}(1)=0}} \int_{0}^{1} R\left(\gamma^{\prime}, J\right) \gamma^{\prime} \cdot J+\left|\nabla_{\gamma^{\prime}} J\right|^{2} d s \\
& =\inf _{\substack{J(0)=T \\
J^{\prime}(1)=0}} \int_{0}^{1} R\left(\gamma^{\prime}, J\right) \gamma^{\prime} \cdot J+\left|\nabla_{\gamma^{\prime}} J\right|^{2} d s \\
& \geq \inf _{\substack{J(0)=T \\
J^{\prime}(1)=0}} \int_{0}^{1} \alpha^{2}\left[\left|\gamma^{\prime}\right|^{2}|J|^{2}-\left(\gamma^{\prime} \cdot J\right)^{2}\right]+\left|J^{\prime}\right|^{2} d s
\end{aligned}
$$

where the sectional curvature of $Y$ is bounded from above by $-\kappa^{-2}$. 
We can, however, compute the last infimum explicitly, since it is achieved by the vector field

$$
\begin{aligned}
J(s)= & \left|\gamma^{\prime}\right|^{-2}\left(\gamma^{\prime} \cdot T\right) \gamma^{\prime}+\left(T-\left|\gamma^{\prime}\right|^{2}\left(\gamma^{\prime} \cdot T\right) \gamma^{\prime}\right) \\
& \cdot\left(\cosh s \kappa^{-1}\left|\gamma^{\prime}\right|-\tanh \kappa^{-1}\left|\gamma^{\prime}\right| \sinh s \kappa^{-1}\left|\gamma^{\prime}\right|\right) .
\end{aligned}
$$

Differentiating this, we get

$$
J^{\prime}(s)=\kappa^{-1}\left|\gamma^{\prime}\right|\left(T-\left|\gamma^{\prime}\right|^{-2}\left(\gamma^{\prime} \cdot T\right) \gamma^{\prime}\right)\left(\sinh s \kappa^{-1}\left|\gamma^{\prime}\right|-\tanh \kappa^{-1}\left|\gamma^{\prime}\right| \cosh s \kappa^{-1}\left|\gamma^{\prime}\right|\right) .
$$

Then

$$
\begin{aligned}
& \left.\int_{0}^{1} \kappa^{-2}\left|\left(\left.\gamma^{\prime}\right|^{-2}|J|^{2}-\left(\gamma^{\prime} \cdot J\right)^{2}\right)+\right| J^{\prime}\right|^{2} d s=\left.J \cdot J^{\prime}\right|_{0} ^{1} \\
& =\kappa^{-1}\left|\gamma^{\prime}\right|\left(\tanh \kappa^{-1}\left|\gamma^{\prime}\right|\right)\left(|T|^{2}-\left|\gamma^{\prime}\right|^{-2}\left(\gamma^{\prime} \cdot T\right)^{2}\right) .
\end{aligned}
$$

We conclude that

Lemma B.1. Given a Riemannian manifold $Y$ with sectional curvature bounded from above by $-\kappa^{-2}<0$, a constant speed geodesic $\gamma:[0,1] \rightarrow Y$, a nonzero tangent vector $T$ at $\gamma(0)$, and a Jacobi field $J$ such that $J(0)=T$,

$$
\int_{0}^{1} R\left(\gamma^{\prime}, J\right) \gamma^{\prime} \cdot J+\left|\nabla_{\gamma^{\prime}} J\right|^{2} d s \geq \kappa^{-1}\left|\gamma^{\prime}\right|\left(\tanh \kappa^{-1}\left|\gamma^{\prime}\right|\right)\left[|T|^{2}-\left|\gamma^{\prime}\right|^{-2}\left(\gamma^{\prime} \cdot T\right)^{2}\right] .
$$

\section{Appendix C. The Douady-Earle extension}

In this section let $B$ denote the unit ball in $\mathbf{R}^{n}$. Let

$$
g=\frac{4|d x|^{2}}{\left(1-|x|^{2}\right)^{2}}
$$

be the standard hyperbolic metric on $B$. Throughout this section, norms denoted $|\cdot|$ and $\|\cdot\|$ are defined with respect to the Euclidean metric, and norms denoted $|\cdot|_{g}$ and $\|\cdot\|_{g}$ are defined with respect to the hyperbolic metric.

Douady and Earle [6] proved the following:

Theorem C.1. Given a homeomorphism $\hat{u}: \partial B \rightarrow \partial B$, there exists a unique map $\mathcal{E}[\hat{u}]=u: B \rightarrow B$ such that for any $\theta \in \partial B$,

$$
\lim _{x \rightarrow \theta} u(x)=\hat{u}(\theta)
$$

and given any $x \in B$,

$$
\int_{\partial B} \phi_{u(x)} \circ \hat{u} \circ \phi_{x}^{-1}(\theta) d \theta=0
$$

where for any $z \in B, \phi_{z}: \bar{B} \rightarrow \bar{B}$ is a Möbius transformation such that $\phi_{z}(z)=0$.

The map $u$ is real analytic. Given any Möbius transformations $\phi, \psi$,

$$
\mathcal{E}[\phi \circ \hat{u} \circ \psi]=\phi \circ \mathcal{E}[\hat{u}] \circ \psi .
$$

Douady and Earle also showed that the value of the extended map and its derivatives at any given point depend continuously on the boundary data. In particular,

Lemma C.2. Given a homeomorphism $\hat{u}_{0}: \partial B \rightarrow \partial B, k>0$, and any $\epsilon>0$, there exists $\delta>0$ such that given any homeomorphism $\hat{u}: \partial B \rightarrow \partial B$ satisfying

$$
\left\|\hat{u}-\hat{u}_{0}\right\|_{\infty}<\delta
$$


we have

$$
\left|\nabla^{k} \mathcal{E}[\hat{u}](0)-\nabla^{k} \mathcal{E}\left[\hat{u}_{0}\right](0)\right|<\epsilon
$$

Lemma C.3. Given $K>1$ and any sequence $\hat{u}_{i}: \partial B \rightarrow \partial B$ of $K$-quasisymmetric maps such that the extended maps $u_{i}=\mathcal{E}\left[\hat{u}_{i}\right]$ satisfy $u_{i}(0)=0$ has a uniformly convergent subsequence.

Proof. Suppose not. Then there exist Möbius transformations $\phi_{i}$ and a subsequence $\hat{u}_{i}$ such that $\phi_{i} \circ \hat{u}_{i}$ does converge uniformly. Therefore, the sequence $\phi_{i} \circ u_{i}(0)$ converges to a limit inside $B$.

On the other hand, no subsequence of $\phi_{i}$ converges. Therefore, there exists a subsequence $\phi_{i}$ such that $\phi_{i} \circ u_{i}(0)=\phi_{i}(0) \rightarrow \partial B$. This is a contradiction.

Corollary C.4. Given $\epsilon>0$, there exists $\delta>0$ such that if $\hat{u}: \partial B \rightarrow \partial B$ is $(1+\delta)$-quasisymmetric, then $u=\mathcal{E}[\hat{u}]$ is a $(1+\epsilon)$-quasi-isometry satisfying

$$
\|\nabla \partial u\|_{g, \infty} \leq \epsilon
$$

Proof. Suppose not. Then for each $k \in \mathbf{Z}^{+}$, there exists a $\left(1+k^{-1}\right)$-quasisymmetric map $\hat{u}_{k}: \partial B \rightarrow \partial B$ and $x_{k} \in D$ such that either

$$
\left|u_{k}^{*} g\left(x_{k}\right)-g\right|_{g}>2 \epsilon
$$

$\mathrm{Or}$

$$
\left|\nabla \partial u_{k}\left(x_{k}\right)\right|_{g}>\epsilon
$$

By composing with Möbius transformations (which are isometries of the metric $g$ ), we may assume that for all $k, x_{k}=u_{k}\left(x_{k}\right)=0$. By Lemma C.3 we may restrict to a subsequence $\hat{u_{k}}$ that converges uniformly to a Möbius transformation. Therefore, by Lemma C.2, $u_{k}^{*} g(0)$ must converge to $g$ and $\nabla \partial u_{k}(0)$ to 0 . This contradicts (C.1) and (C.2).

In dimension 2 Douady-Earle prove more. They show that if the boundary map is quasisymmetric, then the extension is always quasiconformal. In particular,

Theorem 8. Given $K>1$, there exists $K^{*} \geq K$ such that if $\hat{u}: S^{1} \rightarrow S^{1}$ is $K$-quasisymmetric, then $\mathcal{E}[\hat{u}]: D \rightarrow D$ is $K^{*}$-quasiconformal.

\section{REFERENCES}

1. J. P. Anderson, Ph. D. dissertation, 1991.

2. Thierry Aubin, Nonlinear Analysis on Manifolds: Monge-Ampère equations, Springer-Verlag, 1982. MR 85j;58002

3. Isaac Chavel, Eigenvalues in Riemannian geometry, Academic Press, 1984. MR 86g:58140

4. R. R. Coifman and S. Semmes, Interpolation of Banach spaces, Perron processes, and YangMills, American Journal of Mathematics 115 (1993), 243-278. MR 95b:46104

5. W.Y. Ding and Y.D. Wang, Harmonic maps of complete noncompact Riemannian manifolds, Internat. J. Math. 2 (1991), 617-633. MR 93a:58047

6. Adrien Douady and Clifford J. Earle, Conformally natural extension of homeomorphisms of the circle, Acta Math. 157 (1986), 23-48. MR 87j:30041

7. Robert Hardt and Michael Wolf, Harmonic extensions of quasiconformal maps to hyperbolic space, Indiana Univ. Math. J. 46 (1997), 155-163. MR 98i:30032

8. W. Jäger and H. Kaul, Uniqueness and stability of harmonic maps, and their Jacobi fields, Manuscripta Math. 28 (1979), 269-291. MR 80j:58030

9. Uniqueness of harmonic mappings and of solutions of elliptic equations on Riemannian manifolds, Math. Ann. 240 (1979), 231-250. MR 80d:58021 
10. J. Jost and H. Karcher, Geometrische Methoden zur Gewinnung von a priori-Schranken für harmonische Abbildungen, Manuscripta Math. 40 (1982), 27-77. MR 84e:58023

11. _ Almost linear functions and a priori estimates for harmonic maps, Global Riemannian Geometry (T.J. Willmore and N.J. Hitchin, eds.), Ellis Horwood Ser. Math. Appl., E. Horwood, Chichester, 1984, pp. 148-155. CMP 16:17

12. Serge Lang, Differential and Riemannian manifolds, Springer-Verlag, 1995. MR 96d:53001

13. H. Omori, Isometric immersions of Riemannian manifolds, J. Math. Soc. Japan 19 (1967), 205-214. MR 35:6101

14. J.H. Sampson, Some properties and applications of harmonic mappings, Ann. Sci. École Norm. Sup. 11 (1978), 211-228. MR 80b:58031

15. R. Schoen and S.T. Yau, Harmonic maps and the topology of stable hypersurfaces and manifolds of non-negative Ricci curvature, Comment. Math. Helv. 51 (1976), 333-341. MR 55:11302

16. Luen-Fai Tam and Tom Y.-H. Wan, On quasiconformal harmonic maps, Pacific J. Math. 182 (1998), 359-383. CMP 98:09

17. Deane Yang, Convergence of Riemannian manifolds with integral bounds on curvature II, Annales de l'École Normale Supérieure 25 (1992), 179-199. MR 93m:53037

18. S.T. Yau, Harmonic functions on complete Riemannian manifolds, Comm. Pure Appl. Math. 28 (1975), 201-228. MR 55:5502

Department of Mathematics, Polytechnic University, Six Metrotech Center, BrookLYN, NEW YORK 11201

E-mail address: yang@math.poly.edu 